\title{
A Novel Scheme for CP-Length Detection and Initial Synchronization for the LTE Downlink
}

\author{
Antonio A. D'Amico, Michele Morelli, Senior Member, IEEE, and Marco Moretti, Member, IEEE
}

\begin{abstract}
In long-term evolution (LTE) systems, there is an option to extend the size of the cyclic-prefix (CP) when the propagation environment is characterized by severe time dispersion. Such a peculiar feature makes the system more resilient against multipath distortions, but inevitably complicates the downlink synchronization task, which cannot be accomplished through conventional techniques devised for a specified CP size.

In this work, we study the problem of $\mathrm{CP}$ length detection in the LTE downlink in the presence of a timing uncertainty and frequency offset. In contrast to previous investigations based on heuristic arguments, our approach relies on the maximum likelihood (ML) estimation criterion. Furthermore, it accounts for the irregular LTE symbol (characterized by a different CP size), which is present in the normal $\mathrm{CP}$ operation mode. The resulting scheme operates in the time-domain and computes a different metric for each possible position of the irregular symbol within the observation window. Due to its flexibility, the proposed ML approach can be applied to any future multicarrier standard for the design of a $\mathrm{CP}$ length detection algorithm, either in the presence or in the absence of irregular symbols.
\end{abstract}

\section{INTRODUCTION}

The Long Term Evolution (LTE) is a wireless communication standard for high-speed mobile cellular transmissions [1]. Compared to $3 \mathrm{G}$ systems, LTE is characterized by increased data throughput, better spectrum utilization and enhanced user mobility [2]. These advantages are obtained by adopting the multicarrier technology as an air interface, which provides improved resilience against multipath distortions and flexible system scalability by proper adjustment of the transmission bandwidth.

At the start-up or during an handover operation, the user equipment (UE) must activate a cell search procedure to acquire correct downlink synchronization and cell identity information (sector ID and group ID) [3]. To accomplish this task, two pilot sequences, known as Primary Synchronization Signal (PSS) and Secondary Synchronization Signal (SSS), are periodically transmitted by the base station (eNodeB) on a dedicated group of subcarriers. In particular, the PSS conveys the sector ID and provides half-frame timing information, while the SSS specifies the group ID and the frame boundary. A pragmatic approach for cell search is based on the following three-stage procedure. Firstly, symbol timing and fractional frequency offset (FFO) are recovered by using the periodicity introduced by the cyclic prefix (CP). Then, the UE detects the position of the PSS and also determines the integer part of the

Antonio A. D’Amico (antonio.damico@unipi.it), Michele Morelli (michele.morellieunipi.it) and Marco Moretti (marco.moretti@unipi.it) are with the Dipartimento di Ingegneria dell'Informazione of the University of Pisa.

This work was supported by the University of Pisa under the PRA 20182019 Research Project CONCEPT. frequency offset. The final step is devoted to SSS detection, which is accomplished by exploiting information on the PSS position retrieved in the previous stage.

The problem of initial timing and frequency synchronization in the LTE downlink has attracted much attention in the last few years and several solutions are currently available. Some of them rely on the classical approach developed by Van de Beek et al. in [6], where the correlation between the CP and its repetition part is used to localize the start of the OFDM symbol and to get the FFO estimate [7], [8]. Unfortunately, this method cannot work satisfactorily when applied to the LTE downlink. The reason is that it requires prior knowledge of the CP length, while in LTE a normal or extended CP can be chosen according to the delay spread of the propagation channel. In the normal $\mathrm{CP}$ mode, the problem is further complicated by the fact that the first OFDM symbol in the time-slot has a longer CP than the other symbols. Since at the start-up the UE does not know which CP type is currently in use, such information should be retrieved during the time and frequency synchronization process.

As an alternative to the Van de Beek estimation method, some authors suggest to recover timing information by locating the PSS position in the downlink frame [9]-[11]. This approach looks for the peak of the time-domain (TD) cross-correlation between the received samples and the locally regenerated PSS, and has the considerable advantage of being insensitive to the CP length. However, since the PSS is transmitted on a subset of dedicated subcarriers, the TD cross-correlation suffers from remarkable interference originated by the information-bearing subcarriers. A further degradation occurs in the presence of severe channel distortions and frequency mismatches.

While TD-based methods for PSS detection can in principle operate without any CP-type information, identification of the SSS must inevitably be done in the frequency-domain (FD) using the post-FFT samples. Since the precise timing of the SSS with respect to PSS varies with the CP type, accurate estimation of the $\mathrm{CP}$ length is required at this stage to determine the correct position of the FFT window on the time axis. One possible solution is presented in [12], where the FFT process is repeated twice to check for the presence of the SSS under the hypotheses of normal or extended CP. However, considering that SSS detection implies the recognition of one out of 168 different sequences, repeating such a multiple test for the two possible SSS positions results into a substantial processing load. To avoid this problem, the authors of [13] present a pre-FFT method which is able to identify the CP type prior to SSS detection. This algorithm assumes that symbol timing has been acquired at an earlier stage and determines the $\mathrm{CP}$ length by applying the Van de Beek correlation algorithm 
for the two CP choices. A similar scheme was proposed in [14] to determine the CP length in the downlink of a wireless metropolitan area network. In [15], two partial correlations between the $\mathrm{CP}$ and its repetition part are evaluated to acquire $\mathrm{CP}$ type information. In the presence of multipath propagation, however, this method provides unsatisfactory performance and its application is not recommended. The joint estimation of symbol timing, FFO and CP length prior to PSS detection is investigated in [16], where the conventional correlation metric of [6] is evaluated for the two possible CP types over multiple OFDM symbols (what is referred to as symbol folding). In practice, the metrics corresponding to the two CP choices are cut in adjacent segments, which are subsequently summed together. Since the main peaks of the cut pieces will be added constructively only when the correct $\mathrm{CP}$ length is hypothesized, the presence of a sharp peak in one of the two folded metrics allows one to recognize the $\mathrm{CP}$ type, while the peak location provides symbol timing information.

In this work, we present a novel scheme for the joint detection of CP size, symbol timing and FFO in the LTE downlink. The proposed method is able to achieve timing synchronization and CP recognition prior to PSS detection by exploiting the redundancy introduced by the $\mathrm{CP}$. This makes it possible to reveal the PSS in the post-FFT domain with increased accuracy with respect to TD-based methods. Compared to previous investigations, our approach is different for a couple of reasons. First, it is based on maximum likelihood (ML) reasoning, while existing schemes only rely on heuristic arguments. This is expected to produce an estimation algorithm with improved synchronization accuracy and detection capability. Second, it explicitly takes into account that, in the normal CP mode, the first OFDM symbol in the LTE slot is preceded by a $\mathrm{CP}$ of different length with respect to the other symbols. As we shall see, handling such a peculiar feature complicates the $\mathrm{CP}$ detection process in terms of an increased computational requirement. To the best of our knowledge, this is the first work where $\mathrm{CP}$ size detection in the presence of a timing uncertainty and frequency offset is investigated through ML methods. Although derived in the LTE downlink context, the proposed scheme is flexible enough to be applied to any possible future multicarrier communication standard specifying adaptive selection of the $\mathrm{CP}$ size, either in the presence or in the absence of irregular symbols.

The rest of the paper is organized as follows. Section II describes the structure of the LTE downlink signal and provides a summary of the synchronization and cell search procedure. After introducing the signal model in Sect. III, we derive the joint estimator of the $\mathrm{CP}$ mode and synchronization parameters in Sect. IV. We present simulation results in Sect. $\mathrm{V}$ and offer some conclusions in Sect. VI.

Notation: Matrices and vectors are denoted by boldface letters, with $\mathbf{I}_{N}$ being the identity matrix of order $N$ and $\mathbf{A}=\operatorname{diag}\{a(n) ; n=1,2, \ldots, N\}$ indicating an $N \times N$ diagonal matrix with entries $a(n)$ along its main diagonal. We use $\mathrm{E}\{\cdot\},(\cdot)^{*},(\cdot)^{T}$ and $(\cdot)^{H}$ for expectation, complex conjugation, transposition and Hermitian transposition, respectively, while $\mathbf{B}^{-1}$ is the inverse of a matrix $\mathbf{B}$. The notation $\|\cdot\|$ stands for the magnitude of a vector and $|\cdot|$ represents the modulus of a complex-valued quantity. Given a vector $\mathbf{v}$, the function $\mathrm{Sz}(\mathbf{v})$ returns the number of elements of $\mathbf{v}$. Finally, we denote by $\tilde{\lambda}$ a trial value of an unknown parameter $\lambda$, while $\hat{\lambda}$ is the corresponding estimate.

\section{SYSTEM DESCRIPTION}

\section{A. Structure of the downlink radio frame}

According to the 3GPP specifications [1], the OFDM technology is adopted in the physical layer of the LTE downlink. The subcarrier distance is fixed to $\Delta f=15 \mathrm{kHz}$, while the DFT size can vary from 128 to 2048 so as to obtain a scalable transmission bandwidth in the range $1.4-20 \mathrm{MHz}$. Data transmission is arranged in radio frames units of length $T_{f}=307200 T_{s}=10 \mathrm{~ms}$, where $T_{s}=1 /(2048 \cdot \Delta f) \simeq 32.55$ ns is the LTE basic time unit. Each frame consists of ten $1 \mathrm{~ms}$ subframes, and each subframe is further partitioned into two slots of length $T_{\text {slot }}=15360 T_{s}=0.5 \mathrm{~ms}$. Fig. 1 illustrates the structure of the downlink time slot. As is seen, two transmission modes can be adopted depending on the prevailing propagation environment. In the first one (normal $\mathrm{CP})$ each slot conveys seven OFDM symbols, where the $\mathrm{CP}$ length is $160 T_{s}$ for the first symbol and $144 T_{s}$ for the remaining symbols. In the second mode (extended $\mathrm{CP}$ ), only six symbols are present in each slot and all of them are preceded by a CP of length $512 T_{s}$.

Two synchronization signals (PSS and SSS) are periodically transmitted to convey cell identity information, specified by the sector ID and cell group ID. They are sent over a set of 73 subcarriers called synchronization subband (SSB) placed symmetrically around DC. Out of the 73 available SSB subcarriers, only 62 are modulated, while the remaining eleven (five placed at each subband boundary and one at DC) are left unfilled. Both PSS and SSS are broadcast every $5 \mathrm{~ms}$ and their position within the radio frame depends on the adopted duplexing mode (TDD or FDD).

\section{B. Synchronization and cell search procedure}

Initial synchronization and cell search is a fundamental downlink procedure by which the UE can successfully connect to the network. Specifically, initial synchronization allows correct timing and frequency alignment of the UE to the received downlink waveform, while initial cell search provides some necessary information about the serving eNodeB. This task is usually broken down into three hierarchical steps. The first one identifies the OFDM symbol boundary, the FFO and the $\mathrm{CP}$ length by cross-correlating the $\mathrm{CP}$ with its repetition part in the time domain. After frequency correction and $\mathrm{CP}$ removal, the received samples are converted in the frequency domain through an FFT operation. The second step is the PSS detection, by which the sector ID is retrieved together with $5 \mathrm{~ms}$ timing information. Upon successful completion of this task, the UE can determine the SSS position in the downlink frame. The final step is devoted to the SSS identification, which provides the cell group ID along with the frame alignment.

A major peculiarity of the LTE downlink with respect to other commercial OFDM-based systems is that CP type 
recognition is required during the initial synchronization step. This operation is needed whenever PSS detection is performed in the frequency domain using the SSB subcarriers, as it allows the correct positioning of the DFT window on the time axis. Knowledge of the CP length is also necessary for the localization of the SSS relative to the detected PSS. In this work, we concentrate on the initial synchronization step and derive a method to jointly recover the CP transmission mode along with timing and frequency misalignments.

\section{SIGNAL MODEL}

According to the LTE specifications, the downlink signal is converted to baseband and sampled with a fixed frequency $f_{s}=1 / T_{s}=30.72 \mathrm{MHz}$. As a result, the useful part of each OFDM symbol contains $N=2048$ samples regardless of the transmission bandwidth. Furthermore, from Fig. 1 we see that the number of CP samples is $L_{2}=512$ in the extended $\mathrm{CP}$ mode, while it is $L_{0}=160$ or $L_{1}=144$ in the normal $\mathrm{CP}$ mode depending on the position of the OFDM symbol in the frame. We denote by $\{x(n)\}$ the received sample stream and let $f_{d}$ be the CFO between the incoming waveform and the local oscillator used for down-conversion. The timing error is modeled as an unknown integer-valued parameter $\theta$, such that $x(\theta)$ is the first CP sample of some received OFDM symbol in the time slot. Observing that the length of an OFDM symbol (with the CP included) is at most equal to $T_{\max }=\left(N+L_{2}\right) T_{s}$, we conclude that the values of $\theta$ can be restricted to the set $I_{\theta}=\left\{0,1,2, \ldots, N+L_{2}-1\right\}$.
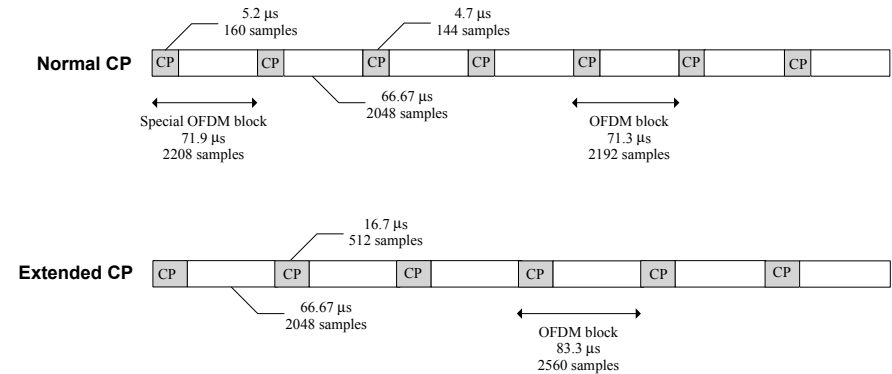

Fig. 1. Structure of the LTE downlink slot: normal and extended CP mode.

As mentioned previously, in the LTE downlink the timing and frequency recovery task is complicated by the fact that, in this initial stage, the UE has no prior knowledge as to whether the normal or extended CP mode is currently in use. Accordingly, traditional correlation-based schemes for OFDM systems available in the literature [6] must be properly modified so as to take such a specific situation into account. Our solution to this problem is inspired by the ML estimation principle and aims at jointly estimating the $\mathrm{CP}$ length and the synchronization parameters. For this purpose, we divide the sequence $\{x(n)\}$ into overlapped segments of length $M=Q\left(N+L_{2}\right)$, where $Q$ is a design parameter belonging to $I_{Q}=\{1,2,3,4,5,6\}$. Each segment corresponds to a different hypothesized value $\tilde{\theta}$ for the timing error and is denoted by $\mathbf{x}_{\tilde{\theta}}=[x(\tilde{\theta}), x(\tilde{\theta}+1), \ldots, x(\tilde{\theta}+M-1)]^{T}$, with $\tilde{\theta} \in I_{\theta}$. The length $M$ is chosen such that $\mathbf{x}_{\tilde{\theta}}$ includes an integer number
$Q$ of OFDM symbols (along with their CP) when the system operates in the extended CP mode. The maximum value of $M$ is attained with $Q=6$ and corresponds to the length of the time slot. On the other hand, when the system operates with a normal $\mathrm{CP}$, each segment encompasses 7 symbols when $Q=6$, and $Q$ symbols plus a residual incomplete symbol when $Q<6$. It can easily be checked that the length of the incomplete symbol is such that it cannot accommodate the copy of its CP for any $Q<6$.

For simplicity, we neglect the impact of multipath distortion on the received waveform. Although this assumption is overoptimistic for a wideband transmission, it has been extensively adopted in the related literature to derive computationally efficient timing recovery schemes for multicarrier transmissions [6]. The entries of $\mathbf{x}(\tilde{\theta})$ are thus modeled as

$$
x(\tilde{\theta}+m)=e^{j 2 \pi(\tilde{\theta}+m) \nu / N} s(\tilde{\theta}+m)+w(\tilde{\theta}+m)
$$

where $\{s(n)\}$ is the discrete-time transmitted signal, $\{w(n)\}$ is additive white Gaussian noise (AWGN) with average power $\sigma_{w}^{2}=\mathrm{E}\left\{|w(n)|^{2}\right\}$ and, finally, $\nu=N T_{s} f_{d}$ is the normalized CFO. The latter is typically decomposed into an integer part plus a remaining FFO $\varepsilon$, which belongs to the interval $[-1 / 2,1 / 2)$.

From the central limit theorem we know that, if the number of modulated subcarriers is sufficiently large, the sample stream $\{s(n)\}$ is approximately Gaussian distributed with zero-mean and power $\sigma_{s}^{2}=\mathrm{E}\left\{|s(n)|^{2}\right\}$. Letting $\mathbf{s}_{\tilde{\theta}}=$ $[s(\tilde{\theta}), s(\tilde{\theta}+1), \ldots, s(\tilde{\theta}+M-1)]^{T}$ and $\mathbf{w}_{\tilde{\theta}}=[w(\tilde{\theta}), w(\tilde{\theta}+$ $1), \ldots, w(\tilde{\theta}+M-1)]^{T}$, we can put (1) in matrix notation as

$$
\mathbf{x}_{\tilde{\theta}}=e^{j 2 \pi \tilde{\theta} \nu / N} \boldsymbol{\Gamma}(\nu) \mathbf{s}_{\tilde{\theta}}+\mathbf{w}_{\tilde{\theta}}
$$

where

$$
\boldsymbol{\Gamma}(\nu)=\operatorname{diag}\left\{e^{j 2 \pi m \nu / N} ; m=0,1, \ldots, M-1\right\} .
$$

In the sequel, vectors $\mathbf{x}_{\tilde{\theta}}$ (with $\tilde{\theta} \in I_{\theta}$ ) are exploited to recover the synchronization parameters $(\theta, \varepsilon)$ and to take a decision in favour of one of the following two hypotheses

$$
\begin{aligned}
\mathcal{H}_{\text {ext }} & : \text { the extended CP mode is adopted } \\
\mathcal{H}_{\text {norm }} & : \text { the normal CP mode is adopted. }
\end{aligned}
$$

\section{ESTIMATION OF THE UNKNOWN PARAMETERS}

Our appoach is inspired by the ML estimation principle and relies on the different statistics of $\mathbf{x}_{\tilde{\theta}}$ conditioned to the alternative hypotheses $\mathcal{H}_{\text {ext }}$ and $\mathcal{H}_{\text {norm }}$. We denote the resulting scheme as the ML-oriented (MLO) algorithm. As we shall see, the MLO computes a set of timing metrics for the hypothesis $\mathcal{H}_{\text {norm }}$, while a single metric is derived for the hypothesis $\mathcal{H}_{\text {ext }}$. All these metrics are subsequently compared to get an estimate of $\theta$ and to detect whether the normal or extended CP is currently in use. The FFO recovery is the last stage of the synchronization procedure.

For each hypothesized value $\tilde{\theta}$ of the timing error, vector $\mathbf{x}_{\tilde{\theta}}$ is assumed to be time aligned with the received sample stream, in the sense that its first entry is regarded as the first CP sample of an OFDM symbol. Since the cases $Q \leq 5$ and $Q=6$ 
lead to a different formulation of the estimation problem, in the sequel we restrict our analysis to $Q \leq 5$ and point out that only marginal modifications are necessary when $Q=6$. Throughout the derivation, the noise and signal powers $\sigma_{w}^{2}$ and $\sigma_{s}^{2}$ are treated as unknown nuisance parameters.

\section{A. Timing metrics under the normal CP hypothesis}

We begin our analysis by assuming that the system operates in the normal CP mode and denote as irregular the first OFDM symbol in the time slot, to emphasize the fact that it is preceded by a longer $\mathrm{CP}$ compared to the other regular symbols. In order to formulate the estimation problem, we must distinguish among $Q+1$ possible alternatives $\mathcal{A}_{\ell}$, which are specified by an index $\ell \in\{0,1, \ldots, Q\}$. In particular, $\ell=0$ corresponds to the absence of the irregular symbol in $\mathbf{x}_{\tilde{\theta}}$, while $1 \leq \ell \leq Q$ indicates that the irregular symbol is present in $\mathbf{x}_{\tilde{\theta}}$ and is preceded by $\ell-1$ regular symbols. In any case, it is convenient to decompose $\mathbf{s}_{\tilde{\theta}}$ in the following way

$$
\mathbf{s}_{\tilde{\theta}}=[\underbrace{\mathbf{u}_{1}^{T} \mathbf{v}_{1}^{T} \mathbf{u}_{1}^{T}}_{1} \underbrace{\mathbf{u}_{2}^{T} \mathbf{v}_{2}^{T} \mathbf{u}_{2}^{T}}_{2} \cdots \underbrace{\mathbf{u}_{Q}^{T} \mathbf{v}_{Q}^{T} \mathbf{u}_{Q}^{T}}_{Q} \overline{\mathbf{z}}_{Q+1}^{T}]^{T}
$$

where the concatenation $\mathbf{z}_{i}^{T}=\left[\mathbf{u}_{i}^{T} \mathbf{v}_{i}^{T} \mathbf{u}_{i}^{T}\right]$ represents the $i$ th OFDM symbol in $\mathbf{s}_{\tilde{\theta}}$ and $\overline{\mathbf{z}}_{Q+1}^{T}$ denotes the residual incomplete symbol. It is worth noting that vector $\mathbf{u}_{i}$ placed in front of $\mathbf{z}_{i}$ corresponds to the $\mathrm{CP}$, while the middle part $\mathbf{v}_{i}$ is the symbol segment that is not copied in the CP. We also observe that $\overline{\mathbf{z}}_{Q+1}^{T}$ is always present whenever $Q \leq 5$, while for $Q=6$ it should be replaced by a complete symbol $\mathbf{z}_{7}^{T}$, thereby leading to a different mathematical model of $\mathbf{s}_{\tilde{\theta}}$. The size of the various segments enclosed in $\mathbf{s}_{\tilde{\theta}}$ depends on the specific alternative $\mathcal{A}_{\ell}$ we are considering. For example, when $\mathcal{A}_{0}$ is assumed to be true, we have

$$
\begin{gathered}
\mathrm{Sz}\left(\mathbf{u}_{i}\right)=L_{1} \\
\mathrm{Sz}\left(\mathbf{v}_{i}\right)=N-L_{1} \\
\mathrm{Sz}\left(\overline{\mathbf{z}}_{Q+1}\right)=Q\left(L_{2}-L_{1}\right)
\end{gathered}
$$

for $i=1,2, \ldots, Q$, while any other alternative $\mathcal{A}_{\ell}$ with $1 \leq$ $\ell \leq Q$ yields

$$
\begin{gathered}
\mathrm{Sz}\left(\mathbf{u}_{i}\right)= \begin{cases}L_{0} & \text { for } i=\ell \\
L_{1} & \text { otherwise }\end{cases} \\
\mathrm{Sz}\left(\mathbf{v}_{i}\right)= \begin{cases}N-L_{0} & \text { for } i=\ell \\
N-L_{1} & \text { otherwise }\end{cases} \\
\mathrm{Sz}\left(\overline{\mathbf{z}}_{Q+1}\right)=Q\left(L_{2}-L_{1}\right)+L_{1}-L_{0} .
\end{gathered}
$$

The fragmentation (4) performed on $\mathbf{s}_{\tilde{\theta}}$ is also applied to the observation vector $\mathbf{x}_{\tilde{\theta}}$. This produces

$$
\mathbf{x}_{\tilde{\theta}}=[\underbrace{\boldsymbol{\alpha}_{1}^{T} \boldsymbol{\xi}_{1}^{T} \boldsymbol{\beta}_{1}^{T}}_{1} \underbrace{\boldsymbol{\alpha}_{2}^{T} \boldsymbol{\xi}_{2}^{T} \boldsymbol{\beta}_{2}^{T}}_{2} \cdots \underbrace{\boldsymbol{\alpha}_{Q}^{T} \boldsymbol{\xi}_{Q}^{T} \boldsymbol{\beta}_{Q}^{T}}_{Q} \overline{\boldsymbol{\xi}}_{Q+1}^{T}]^{T}
$$

where each segment has the same length of the corresponding segment in $\mathbf{s}_{\tilde{\theta}}$, i.e.,

$$
\begin{gathered}
\mathrm{Sz}\left(\boldsymbol{\alpha}_{i}\right)=\operatorname{Sz}\left(\boldsymbol{\beta}_{i}\right)=\operatorname{Sz}\left(\mathbf{u}_{i}\right) \\
\operatorname{Sz}\left(\boldsymbol{\xi}_{i}\right)=\operatorname{Sz}\left(\mathbf{v}_{i}\right) \\
\operatorname{Sz}\left(\overline{\boldsymbol{\xi}}_{Q+1}\right)=\operatorname{Sz}\left(\overline{\mathbf{z}}_{Q+1}\right)
\end{gathered}
$$

for $i=1,2, \ldots, Q$. Comparing (4) and (7), it turns out that $\left[\boldsymbol{\alpha}_{i}^{T} \boldsymbol{\xi}_{i}^{T} \boldsymbol{\beta}_{i}^{T}\right]$ represents the received version of the $i$ th OFDM symbol $\left[\mathbf{u}_{i}^{T} \mathbf{v}_{i}^{T} \mathbf{u}_{i}^{T}\right]$, where $\boldsymbol{\beta}_{i}$ differs from $\boldsymbol{\alpha}_{i}$ as a consequence of the noise contribution and the phase rotation induced by the CFO.

In order to apply the ML estimation criterion, we must determine the probability density function (pdf) of $\mathbf{x}_{\tilde{\theta}}$ for each possible alternative $\mathcal{A}_{\ell}$. Recalling that $\mathbf{s}_{\tilde{\theta}}$ and $\mathbf{w}_{\tilde{\theta}}$ are statistically independent zero-mean Gaussian vectors, from (2) it follows that $\mathbf{x}_{\tilde{\theta}}$ is Gaussian distributed as well, with zero mean and covariance matrix

$$
\mathbf{C}_{x}=\boldsymbol{\Gamma}(\nu) \mathbf{C}_{s} \boldsymbol{\Gamma}^{H}(\nu)+\mathbf{C}_{w}
$$

where $\mathbf{C}_{s}=\mathrm{E}\left\{\mathbf{s}_{\tilde{\theta}} \mathbf{s}_{\tilde{\theta}}^{T}\right\}$ and $\mathbf{C}_{w}=\mathrm{E}\left\{\mathbf{w}_{\tilde{\theta}} \mathbf{w}_{\tilde{\theta}}^{H}\right\}=\sigma_{w}^{2} \mathbf{I}_{M}$. Its pdf is thus given by [17]

$$
\begin{aligned}
f\left(\mathbf{x}_{\tilde{\theta}}\right)= & \frac{1}{\pi^{M} \operatorname{det}\left(\mathbf{C}_{s}+\sigma_{w}^{2} \mathbf{I}_{M}\right)} \\
& \times \exp \left[-\mathbf{x}_{\tilde{\theta}}^{H} \boldsymbol{\Gamma}(\nu)\left(\mathbf{C}_{s}+\sigma_{w}^{2} \mathbf{I}_{M}\right)^{-1} \boldsymbol{\Gamma}^{H}(\nu) \mathbf{x}_{\tilde{\theta}}\right] .
\end{aligned}
$$

Since the received signal is sampled at a fixed frequency $f_{s}=2048 \cdot \Delta f$ while the maximum number of modulated subcarriers is 1200 , it can easily be shown that adjacent entries of $\mathbf{s}_{\tilde{\theta}}$ are statistically correlated. This fact considerably complicates the estimation problem, if only because the correlation coefficient depends on the transmission bandwidth, which is unknown at this stage. For the sake of simplicity, we suggest to ignore such a correlation. In particular, we assume that all the subvectors present in the right-hand-side of (4) are statistically independent from each other and have independent entries with average power $\sigma_{s}^{2}$. This amounts to assuming

$$
\mathbf{C}_{s}=\operatorname{diag}\left\{\mathbf{C}_{z_{1}}, \mathbf{C}_{z_{2}}, \ldots, \mathbf{C}_{z_{Q}}, \mathbf{C}_{\bar{z}_{Q+1}}\right\}
$$

where $\mathbf{C}_{\bar{z}_{Q+1}}=\mathrm{E}\left\{\overline{\mathbf{z}}_{Q+1} \overline{\mathbf{z}}_{Q+1}^{H}\right\}=\sigma_{s}^{2} \mathbf{I}_{\mathrm{Sz}\left(\overline{\mathbf{z}}_{Q+1}\right)}$ and

$$
\mathbf{C}_{z_{i}}=\mathrm{E}\left\{\mathbf{z}_{i} \mathbf{z}_{i}^{H}\right\}=\sigma_{s}^{2}\left[\begin{array}{ccc}
\mathbf{I}_{\mathrm{Sz}\left(\mathbf{u}_{i}\right)} & \mathbf{0} & \mathbf{I}_{\mathrm{Sz}\left(\mathbf{u}_{i}\right)} \\
\mathbf{0} & \mathbf{I}_{\mathrm{Sz}\left(\mathbf{v}_{i}\right)} & \mathbf{0} \\
\mathbf{I}_{\mathrm{Sz}\left(\mathbf{u}_{i}\right)} & \mathbf{0} & \mathbf{I}_{\mathrm{Sz}\left(\mathbf{u}_{i}\right)}
\end{array}\right] .
$$

The true statistics of $\mathbf{s}_{\tilde{\theta}}$ will be used in the numerical analysis to assess their impact on the system performance.

To proceed further, we call $\sigma^{2}=\sigma_{s}^{2}+\sigma_{w}^{2}$ the power of $x(\tilde{\theta}+m)$ and let $\gamma=\sigma_{s}^{2} / \sigma^{2}$. Furthermore, we denote by $f\left(\mathbf{x}_{\tilde{\theta}} \mid \tilde{\ell} ; \tilde{\gamma}, \tilde{\sigma}^{2}, \tilde{\varepsilon}\right)$ the conditional pdf of $\mathbf{x}_{\tilde{\theta}}$ given $\mathcal{A}_{\tilde{\ell}}$ $(\tilde{\ell}=0,1, \ldots, Q)$ and the set $\left(\tilde{\gamma}, \tilde{\sigma}^{2}, \tilde{\varepsilon}\right)$ of unknown parameters. Substituting (11) and (12) into (10), after standard manipulations we get

$$
\begin{aligned}
& f\left(\mathbf{x}_{\tilde{\theta}} \mid \tilde{\ell} ; \tilde{\gamma}, \tilde{\sigma}^{2}, \tilde{\varepsilon}\right)=\frac{1}{\pi^{M} \tilde{\sigma}^{2 M}\left(1-\tilde{\gamma}^{2}\right)^{d_{1}}} \\
& \quad \times \exp \left\{-\frac{1}{\tilde{\sigma}^{2}}\left[a_{1}+\frac{b_{1}-2 c_{1} \tilde{\gamma} \cos \left(2 \pi \tilde{\varepsilon}-\varphi_{1}\right)}{1-\tilde{\gamma}^{2}}\right]\right\}
\end{aligned}
$$


where we have defined the quantities

$$
\begin{aligned}
& a_{1}(\tilde{\theta}, \tilde{\ell})=\left\|\overline{\boldsymbol{\xi}}_{Q+1}\right\|^{2}+\sum_{i=1}^{Q}\left\|\boldsymbol{\xi}_{i}\right\|^{2} \\
& b_{1}(\tilde{\theta}, \tilde{\ell})=\sum_{i=1}^{Q}\left(\left\|\boldsymbol{\alpha}_{i}\right\|^{2}+\left\|\boldsymbol{\beta}_{i}\right\|^{2}\right) \\
& c_{1}(\tilde{\theta}, \tilde{\ell})=\left|\sum_{i=1}^{Q} \boldsymbol{\alpha}_{i}^{H} \boldsymbol{\beta}_{i}\right| \\
& \varphi_{1}(\tilde{\theta}, \tilde{\ell})=\arg \left\{\sum_{i=1}^{Q} \boldsymbol{\alpha}_{i}^{H} \boldsymbol{\beta}_{i}\right\} \\
& d_{1}(\tilde{\ell})= \begin{cases}Q L_{1} & \text { for } \tilde{\ell}=0 \\
L_{0}+(Q-1) L_{1} & \text { for } \tilde{\ell} \neq 0\end{cases}
\end{aligned}
$$

and we have explicitly indicated their dependence on $\tilde{\theta}$ (through $\mathbf{x}_{\tilde{\theta}}$ ) and $\tilde{\ell}$ (through the size of the various vectors appearing in (14)).

For a given observation vector $\mathbf{x}_{\tilde{\theta}}$ and for any value of $\tilde{\ell} \in\{0,1, \ldots, Q\}$, the joint ML estimate of $\left(\gamma, \sigma^{2}, \varepsilon\right)$ is the location where $f\left(\mathbf{x}_{\tilde{\theta}} \mid \tilde{\ell} ; \tilde{\gamma}, \tilde{\sigma}^{2}, \tilde{\varepsilon}\right)$ achieves its global maximum. The latter can be found through the following three-step procedure. In the first step, we keep $\tilde{\gamma}$ and $\tilde{\sigma}^{2}$ fixed and let $\tilde{\varepsilon}$ vary. In such a case, the maximum of (13) is achieved for

$$
\hat{\varepsilon}\left(\mathbf{x}_{\tilde{\theta}} \mid \tilde{\ell}\right)=\frac{1}{2 \pi} \varphi_{1}(\tilde{\theta}, \tilde{\ell})
$$

and takes the value

$$
\begin{aligned}
f\left(\mathbf{x}_{\tilde{\theta}} \mid \tilde{\ell} ; \tilde{\gamma}, \tilde{\sigma}^{2}\right)= & \frac{1}{\pi^{M} \tilde{\sigma}^{2 M}\left(1-\tilde{\gamma}^{2}\right)^{d_{1}}} \\
& \times \exp \left\{-\frac{1}{\tilde{\sigma}^{2}}\left(a_{1}+\frac{b_{1}-2 \tilde{\gamma} c_{1}}{1-\tilde{\gamma}^{2}}\right)\right\} .
\end{aligned}
$$

In the second step, we fix $\tilde{\gamma}$ and maximize (16) with respect to $\tilde{\sigma}^{2}$. This produces

$$
\hat{\sigma}^{2}\left(\mathbf{x}_{\tilde{\theta}} \mid \tilde{\ell} ; \tilde{\gamma}\right)=\frac{1}{M}\left(a_{1}+\frac{b_{1}-2 \tilde{\gamma} c_{1}}{1-\tilde{\gamma}^{2}}\right)
$$

which, after substitution into (16), leads to

$$
f\left(\mathbf{x}_{\tilde{\theta}} \mid \tilde{\ell} ; \tilde{\gamma}\right)=\frac{\left(M e^{-1} / \pi\right)^{M}}{\left(a_{1}+\frac{b_{1}-2 c_{1} \tilde{\gamma}}{1-\tilde{\gamma}^{2}}\right)^{M}\left(1-\tilde{\gamma}^{2}\right)^{d_{1}}}
$$

or, equivalently,

$$
f\left(\mathbf{x}_{\tilde{\theta}} \mid \tilde{\ell} ; \tilde{\gamma}\right)=\frac{\left(M e^{-1} / \pi\right)^{M}\left(1-\tilde{\gamma}^{2}\right)^{M-d_{1}}}{\left\|\mathbf{x}_{\tilde{\theta}}\right\|^{2 M}\left(1-\frac{a_{1} \tilde{\gamma}^{2}+2 c_{1} \tilde{\gamma}}{\left\|\mathbf{x}_{\tilde{\theta}}\right\|^{2}}\right)^{M}}
$$

where we have use the identity $a_{1}+b_{1}=\left\|\mathbf{x}_{\tilde{\theta}}\right\|^{2}$. Observing that $\left\|\mathbf{x}_{\tilde{\theta}}\right\|^{2}$ is independent of $\tilde{\gamma}$ and it is also asymptotically (i.e., when $M$ goes to infinity) independent of $\tilde{\theta}$, we can reasonably neglect the multiplicative term $\left[M e^{-1} /\left(\pi\left\|\mathbf{x}_{\tilde{\theta}}\right\|^{2}\right)\right]^{M}$ in (19). Hence, after applying the logarithmic function, we replace $f\left(\mathbf{x}_{\tilde{\theta}} \mid \tilde{\ell} ; \tilde{\gamma}\right)$ by

$\Phi\left(\mathbf{x}_{\tilde{\theta}} \mid \tilde{\ell} ; \tilde{\gamma}\right)=-M \ln \left(1-\frac{a_{1} \tilde{\gamma}^{2}+2 c_{1} \tilde{\gamma}}{\left\|\mathbf{x}_{\tilde{\theta}}\right\|^{2}}\right)+\left(M-d_{1}\right) \ln \left(1-\tilde{\gamma}^{2}\right)$.

The final step looks for the maximum of $\Phi\left(\mathbf{x}_{\tilde{\theta}} \mid \tilde{\ell} ; \tilde{\gamma}\right)$. For this purpose, we set to zero the derivate of (20) with respect to $\tilde{\gamma}$ and obtain the third degree polynomial equation $P_{1}(\tilde{\gamma})=0$, where

$$
\begin{aligned}
P_{1}(\tilde{\gamma})= & d_{1} a_{1} \tilde{\gamma}^{3}-\left(M-2 d_{1}\right) c_{1}^{2} \tilde{\gamma} \\
& -\left[M a_{1}-\left(M-d_{1}\right)\left\|\mathbf{x}_{\tilde{\theta}}\right\|^{2}\right] \tilde{\gamma}-M c_{1} .
\end{aligned}
$$

Since such equation may have up to three different solutions, the question arises as how to identify the solution that provides the best estimate of $\gamma$. One possible approach relies on the fact that $P_{1}(0)=-M c_{1}<0$ and $P_{1}(1)=b_{1}-2 c_{1}>0$, so that at least one root of $P_{1}(\tilde{\gamma})$ belongs to the interval $I_{\gamma}=[0,1]$. Hence, observing that $\gamma=\sigma_{s}^{2} /\left(\sigma_{s}^{2}+\sigma_{w}^{2}\right) \in I_{\gamma}$, we suggest to discard any root outside $I_{\gamma}$ and, in the event that two or three roots are found in $I_{\gamma}$, we select the one for which $\Phi\left(\mathbf{x}_{\tilde{\theta}} \mid \tilde{\ell} ; \tilde{\gamma}\right)$ is maximum. The selected root, say $\hat{\gamma}_{1}(\tilde{\theta}, \tilde{\ell})$, is eventually substituted into (20). This provides the timing metrics for the hypothesis $\mathcal{H}_{\text {norm }}$ in the form

$$
\begin{aligned}
\lambda_{1}(\tilde{\theta}, \tilde{\ell})= & -M \ln \left[1-\frac{a_{1} \hat{\gamma}_{1}^{2}(\tilde{\theta}, \tilde{\ell})+2 c_{1} \hat{\gamma}_{1}(\tilde{\theta}, \tilde{\ell})}{\left\|\mathbf{x}_{\tilde{\theta}}\right\|^{2}}\right] \\
& +\left(M-d_{1}\right) \ln \left[1-\hat{\gamma}_{1}^{2}(\tilde{\theta}, \tilde{\ell})\right] .
\end{aligned}
$$

\section{$B$. Timing metric under the extended $C P$ hypothesis}

We now assume that the system operates in the extended $\mathrm{CP}$ mode and derive the corresponding timing metric based on the observation vector $\mathbf{x}_{\tilde{\theta}}$. The procedure is essentially the same illustrated under the normal CP hypothesis with some minor adjustments. The first one arises from the fact that $\mathbf{s}_{\tilde{\theta}}$ includes an integer number $Q$ of OFDM symbols, so that the incomplete symbol $\overline{\mathbf{z}}_{Q+1}^{T}$ no longer appears in (4). The second adjustment is related to the absence of the irregular OFDM symbol in the time slot, which reduces the $Q+1$ alternatives $\mathcal{A}_{\ell}$ of the normal CP mode to a single instance $\mathcal{A}_{0}$. Putting these facts together, we decompose $\mathbf{s}_{\tilde{\theta}}$ and $\mathbf{x}_{\tilde{\theta}}$ as

$$
\begin{gathered}
\mathbf{s}_{\tilde{\theta}}=[\underbrace{\mathbf{u}_{1}^{T} \mathbf{v}_{1}^{T} \mathbf{u}_{1}^{T}}_{1} \underbrace{\mathbf{u}_{2}^{T} \mathbf{v}_{2}^{T} \mathbf{u}_{2}^{T}}_{2} \cdots \underbrace{\mathbf{u}_{Q}^{T} \mathbf{v}_{Q}^{T} \mathbf{u}_{Q}^{T}}_{Q}]^{T} \\
\mathbf{x}_{\tilde{\theta}}=[\underbrace{\boldsymbol{\alpha}_{1}^{T} \boldsymbol{\xi}_{1}^{T} \boldsymbol{\beta}_{1}^{T}}_{1} \underbrace{\boldsymbol{\alpha}_{2}^{T} \boldsymbol{\xi}_{2}^{T} \boldsymbol{\beta}_{2}^{T}}_{2} \cdots \underbrace{\boldsymbol{\alpha}_{Q}^{T} \boldsymbol{\xi}_{Q}^{T} \boldsymbol{\beta}_{Q}^{T}}_{Q}]^{T}
\end{gathered}
$$

with

$$
\begin{gathered}
\operatorname{Sz}\left(\boldsymbol{\alpha}_{i}\right)=\operatorname{Sz}\left(\boldsymbol{\beta}_{i}\right)=\operatorname{Sz}\left(\mathbf{u}_{i}\right)=L_{2} \\
\operatorname{Sz}\left(\boldsymbol{\xi}_{i}\right)=\operatorname{Sz}\left(\mathbf{v}_{i}\right)=N-L_{2} .
\end{gathered}
$$

The pdf of $\mathbf{x}_{\tilde{\theta}}$ is expressed by

$$
\begin{aligned}
& f\left(\mathbf{x}_{\tilde{\theta}} \mid \tilde{\gamma}, \tilde{\sigma}^{2}, \tilde{\varepsilon}\right)=\frac{1}{\pi^{M} \tilde{\sigma}^{2 M}\left(1-\tilde{\gamma}^{2}\right)^{d_{2}}} \\
& \quad \times \exp \left\{-\frac{1}{\tilde{\sigma}^{2}}\left[a_{2}+\frac{b_{2}-2 c_{2} \tilde{\gamma} \cos \left(2 \pi \tilde{\varepsilon}-\varphi_{2}\right)}{1-\tilde{\gamma}^{2}}\right]\right\}
\end{aligned}
$$


where $d_{2}=Q L_{2}$ and

$$
a_{2}(\tilde{\theta})=\sum_{i=1}^{Q}\left\|\boldsymbol{\xi}_{i}\right\|^{2} .
$$

As for the quantities $\left\{b_{2}, c_{2}, \varphi_{2}\right\}$, they depend on $\tilde{\theta}$ through $\mathbf{x}_{\tilde{\theta}}$ and have the same expression of $\left\{b_{1}, c_{1}, \varphi_{1}\right\}$ given in (14), the only difference being in the size of the various subvectors.

The maximum of the pdf over the set of parameters $\left\{\tilde{\gamma}, \tilde{\sigma}^{2}, \tilde{\epsilon}\right\}$ is found by applying the same procedure illustrated under the $\mathcal{H}_{\text {norm }}$ hypothesis. This produces the timing metric

$$
\begin{aligned}
\lambda_{2}(\tilde{\theta})= & -M \ln \left[1-\frac{a_{2} \hat{\gamma}_{2}^{2}(\tilde{\theta})+2 c_{2} \hat{\gamma}_{2}(\tilde{\theta})}{\left\|\mathbf{x}_{\tilde{\theta}}\right\|^{2}}\right] \\
& +\left(M-d_{2}\right) \ln \left[1-\hat{\gamma}_{2}^{2}(\tilde{\theta})\right]
\end{aligned}
$$

where $\hat{\gamma}_{2}(\tilde{\theta})$ is the root of the polynomial

$$
\begin{aligned}
P_{2}(\tilde{\gamma})= & d_{2} a_{2} \tilde{\gamma}^{3}-\left(M-2 d_{2}\right) c_{2}^{2} \tilde{\gamma} \\
& -\left[M a_{2}-\left(M-d_{2}\right)\left\|\mathbf{x}_{\tilde{\theta}}\right\|^{2}\right] \tilde{\gamma}-M c_{2}
\end{aligned}
$$

belonging to $I_{\gamma}$, which is selected as indicated previously under the $\mathcal{H}_{\text {norm }}$ hypothesis.

\section{Estimation of the CP mode and synchronization parameters with $M L O$}

The timing metrics shown in (22) and (27) are now exploited to detect the employed CP mode and to retrieve the synchronization parameters $(\theta, \varepsilon)$. For this purpose, we suggest the following three-step procedure:

Step 1) Compute the quantities

$$
\begin{gathered}
\left(\hat{\theta}_{\text {norm }}, \hat{\ell}\right)=\arg \max _{(\tilde{\theta}, \tilde{l})}\left\{\lambda_{1}(\tilde{\theta}, \tilde{\ell})\right\} \\
\hat{\theta}_{\text {ext }}=\arg \max _{\tilde{\theta}}\left\{\lambda_{2}(\tilde{\theta})\right\}
\end{gathered}
$$

where $\hat{\ell}$ identifies one of the alternatives $\mathcal{A}_{\ell}$ under the hypothesis $\mathcal{H}_{\text {norm }}$, while $\hat{\theta}_{\text {norm }}$ and $\hat{\theta}_{\text {ext }}$ are the timing estimates conditioned on $\mathcal{H}_{\text {norm }}$ and $\mathcal{H}_{\text {ext }}$, respectively.

Step 2) Compare $\lambda_{1}\left(\hat{\theta}_{\text {norm }}, \hat{\ell}\right)$ with $\lambda_{2}\left(\hat{\theta}_{\text {ext }}\right)$ and apply the decision rule

$$
\lambda_{1}\left(\hat{\theta}_{\text {norm }}, \hat{\ell}\right) \underset{\mathcal{H}_{\text {ext }}}{\stackrel{\mathcal{H}_{\text {norm }}}{\gtrless}} \lambda_{2}\left(\hat{\theta}_{\text {ext }}\right) .
$$

Step 3) Obtain the timing and frequency estimates as

$$
\begin{gathered}
\left\{\begin{array}{c}
\hat{\theta}=\hat{\theta}_{\text {norm }} \\
\hat{\varepsilon}=\varphi_{1}\left(\hat{\theta}_{\text {norm }}, \hat{\ell}\right) /(2 \pi)
\end{array} \quad \text { if decided in favour of } \mathcal{H}_{\text {norm }}\right. \\
\left\{\begin{array}{c}
\hat{\theta}=\hat{\theta}_{\text {ext }} \\
\hat{\varepsilon}=\varphi_{2}\left(\hat{\theta}_{\text {ext }}\right) /(2 \pi)
\end{array}\right.
\end{gathered}
$$

\section{Iterative computation of the timing metrics and complexity analysis}

The timing metrics $\lambda_{1}(\tilde{\theta}, \tilde{\ell})$ and $\lambda_{2}(\tilde{\theta})$ shown in (22) and (27) are efficiently computed by updating $\left\|\mathbf{x}_{\tilde{\theta}}\right\|^{2}, a_{1}(\tilde{\theta}, \tilde{\ell})$, $a_{2}(\tilde{\theta}), c_{1}(\tilde{\theta}, \tilde{\ell})$ and $c_{2}(\tilde{\theta})$ at each new received sample. To understand how it comes about and to evaluate the computational complexity of MLO, we start by observing that the sequences $|x(n)|^{2}$ and $x(n) x^{*}(n-N)$ are the constituent terms of all the aforementioned quantities, and hence it is convenient to store them into two shift-registers of length $M$. Recalling that a complex multiplication is equivalent to four real multiplications plus two real additions, the registers are updated at each new sampling period with a total of 9 floating point operations (flops). Their content is subsequently used to evaluate all the terms appearing in the expressions of the MLO metrics as shown in the sequel.

1) Iterative computation of $\left\|\mathrm{x}_{\tilde{\theta}}\right\|^{2}$ : We can obtain $\left\|\mathrm{x}_{\tilde{\theta}}\right\|^{2}$ from $\left\|\mathbf{x}_{\tilde{\theta}-1}\right\|^{2}$ through the following iterative equation

$$
\left\|\mathbf{x}_{\tilde{\theta}}\right\|^{2}=\left\|\mathbf{x}_{\tilde{\theta}-1}\right\|^{2}+|x(\tilde{\theta}+M-1)|^{2}-|x(\tilde{\theta}-1)|^{2},
$$

which requires 2 flops.

2) Iterative computation of $a_{1}(\tilde{\theta}, \tilde{\ell})$ : From (14) and (7), it follows that $a_{1}(\tilde{\theta}, \tilde{\ell})$ can be computed as

$$
a_{1}(\tilde{\theta}, \tilde{\ell})=a_{1}(\tilde{\theta}-1, \tilde{\ell})+\sum_{i=1}^{Q+1} \delta_{i}(\tilde{\ell})
$$

where

$$
\begin{aligned}
& \delta_{i}(\tilde{\ell})=\left\|\boldsymbol{\xi}_{i}(\tilde{\theta}, \tilde{\ell})\right\|^{2}-\left\|\boldsymbol{\xi}_{i}(\tilde{\theta}-1, \tilde{\ell})\right\|^{2} \quad i=1,2, \ldots, Q \\
& \delta_{Q+1}(\tilde{\ell})=\left\|\overline{\boldsymbol{\xi}}_{Q+1}(\tilde{\theta}, \tilde{\ell})\right\|^{2}-\left\|\overline{\boldsymbol{\xi}}_{Q+1}(\tilde{\theta}-1, \tilde{\ell})\right\|^{2}
\end{aligned}
$$

and we have explicitly indicated the dependence of vectors $\boldsymbol{\xi}_{i}$ on $\tilde{\theta}$ and $\tilde{\ell}$. Each $\delta_{i}(\tilde{\ell})$ can be written as

$$
\delta_{i}(\tilde{\ell})=\left|x\left(\tilde{\theta}+k_{i}^{\prime}(\tilde{\ell})\right)\right|^{2}-\left|x\left(\tilde{\theta}-1+k_{i}^{\prime \prime}(\tilde{\ell})\right)\right|^{2}
$$

where $k_{i}^{\prime}(\tilde{\ell})$ and $k_{i}^{\prime \prime}(\tilde{\ell})$ depend on $i$ and $\tilde{\ell}$. For example, $k_{i}^{\prime}(\tilde{\ell})=$ $N-1$ and $k_{i}^{\prime \prime}(\tilde{\ell})=L_{1}$ for $i=1$ and $\tilde{\ell}=0$. In addition, it can be shown that

$$
\begin{array}{ll}
\delta_{i}(\tilde{\ell})=\delta_{i}(0) & \text { for } \tilde{\ell}=i+1, i+2, \ldots, Q \\
\delta_{i}(\tilde{\ell})=\delta_{i}(1) & \text { for } \tilde{\ell}=2,3, \ldots, i-1
\end{array}
$$

This means that, for $\tilde{\ell}=2,3, \ldots, Q$, the updating term in (33) can be written as follows

$$
\sum_{i=1}^{Q+1} \delta_{i}(\tilde{\ell})=\sum_{i=1}^{\tilde{\ell}-1} \delta_{i}(0)+\delta_{\tilde{\ell}}(\tilde{\ell})+\sum_{i=\tilde{\ell}+1}^{Q+1} \delta_{i}(1)
$$

and hence, for implementing (33), we only need $\left\{\sum_{i=1}^{k} \delta_{i}(\tilde{\ell}) ; k=1,2, \ldots, Q+1\right.$ and $\left.\tilde{\ell}=0,1\right\} \quad$ and $\left\{\delta_{\tilde{\ell}}(\tilde{\ell}) ; \tilde{\ell}=2,3, \ldots, Q\right\}$. Taking (33), (35) and (37) into account, it turns out that the computation of $\left\{a_{1}(\tilde{\theta}, \tilde{\ell}) ; \tilde{\ell}=0,1, \ldots, Q\right\}$ requires a total of $8 Q$ flops.

3) Iterative computation of $c_{1}(\tilde{\theta}, \tilde{\ell})$ : We first consider the recursive computation of $\breve{c}_{1}(\tilde{\theta}, \tilde{\ell})=\sum_{i=1}^{Q} \boldsymbol{\alpha}_{i}^{H}(\tilde{\theta}, \tilde{\ell}) \boldsymbol{\beta}_{i}(\tilde{\theta}, \tilde{\ell})$. From (14) and (7) one easily gets

$$
\breve{c}_{1}(\tilde{\theta}, \tilde{\ell})=\breve{c}_{1}(\tilde{\theta}-1, \tilde{\ell})+\sum_{i=1}^{Q} \delta_{i}^{\prime}(\tilde{\ell})
$$

where

$$
\delta_{i}^{\prime}(\tilde{\ell})=\boldsymbol{\alpha}_{i}^{H}(\tilde{\theta}, \tilde{\ell}) \boldsymbol{\beta}_{i}(\tilde{\theta}, \tilde{\ell})-\boldsymbol{\alpha}_{i}^{H}(\tilde{\theta}-1, \tilde{\ell}) \boldsymbol{\beta}_{i}(\tilde{\theta}-1, \tilde{\ell}) .
$$


We can rewrite $\delta_{i}^{\prime}(\tilde{\ell})$ as

$$
\begin{aligned}
\delta_{i}^{\prime}(\tilde{\ell})= & x^{*}\left(\tilde{\theta}+\kappa_{i}^{\prime}(\tilde{\ell})\right) x\left(\tilde{\theta}+\kappa_{i}^{\prime}(\tilde{\ell})+N\right) \\
& -x^{*}\left(\tilde{\theta}-1+\kappa_{i}^{\prime \prime}(\tilde{\ell})\right) x\left(\tilde{\theta}-1+\kappa_{i}^{\prime \prime}(\tilde{\ell})+N\right)
\end{aligned}
$$

where $\kappa_{i}^{\prime}(\tilde{\ell})$ and $\kappa_{i}^{\prime \prime}(\tilde{\ell})$ depend on $i$ and $\tilde{\ell}$. For example, $\kappa_{i}^{\prime}(\tilde{\ell})=L_{1}-1$ and $\kappa_{i}^{\prime \prime}(\tilde{\ell})=0$ for $i=1$ and $\tilde{\ell}=0$. After a careful analysis, by using the same arguments as for the computation of $a_{1}(\tilde{\theta}, \tilde{\ell})$, it can be proved that a total of $2(8 Q-5)$ real additions are required for computing $\left\{\breve{c}_{1}(\tilde{\theta}, \tilde{\ell}) ; \tilde{\ell}=0,1, \ldots, Q\right\}$. Observing that a modulus evaluation (3 flops) is used to get $c_{1}(\tilde{\theta}, \tilde{\ell})$ from $\breve{c}_{1}(\tilde{\theta}, \tilde{\ell})$, it turns out that the computation of $\left\{c_{1}(\tilde{\theta}, \tilde{\ell}) ; \tilde{\ell}=0,1, \ldots, Q\right\}$ requires a total of $19 Q-7$ flops.

Once $a_{1}(\tilde{\theta}, \tilde{\ell})$ and $c_{1}(\tilde{\theta}, \tilde{\ell})$ are available, the coefficients of the polynomial $P_{1}(\tilde{\gamma})$ in $(21)$ are evaluated with $6(Q+1)$ flops, and the metrics $\lambda_{1}(\tilde{\theta}, \tilde{\ell})$ are eventually obtained with $9(Q+$ 1 ) additional flops. Collecting the above results, we conclude that the processing load of MLO under the hypothesis $\mathcal{H}_{\text {norm }}$ amounts to $42 Q+19$ flops for each new received sample.

To assess the complexity under the hypothesis $\mathcal{H}_{\text {ext }}$, we observe that $2 Q$ real additions plus $2 Q$ complex additions and one modulus evaluation are required to update $a_{2}(\tilde{\theta})$ and $c_{2}(\tilde{\theta})$. These quantities are then used to compute the coefficients of the polynomial $P_{2}(\tilde{\gamma})$ in (28) with 6 flops. Since 9 further flops are needed to evaluate the metric $\lambda_{2}(\tilde{\theta})$, the processing requirement is quantified at $6 Q+18$ flops. Summing this result to the correspondent value found under the hypothesis $\mathcal{H}_{\text {norm }}$, yields an overall complexity of $48 Q+37$ flops for the MLO.

\section{E. Application of $M L O$ in the absence of the irregular OFDM symbol}

Although specifically designed for the 3GPP LTE downlink, the MLO is a flexible scheme which, after some minor modifications, can be applied to any OFDM communication system where the $\mathrm{CP}$ size is adaptively selected from a given set depending on the actual propagation environment. To fix ideas, assume that no irregular symbol is specified in the standard and only two CP sizes $L_{1}$ (normal CP) and $L_{2}>L_{1}$ (extended CP) are available. Then, a single metric $\lambda_{1}(\tilde{\theta})$ is computed under the hypothesis $\mathcal{H}_{\text {norm }}$ by simply putting $\tilde{\ell}$ $=0$ in the expression of $\lambda_{1}(\tilde{\theta}, \tilde{\ell})$ in (22), while the metric $\lambda_{2}(\tilde{\theta})$ under the hypothesis $\mathcal{H}_{\text {ext }}$ is exactly the same as that shown in (27). A decision on the adopted CP size is next taken according to the following rule

$$
\lambda_{1}\left(\hat{\theta}_{\text {norm }}\right) \stackrel{\mathcal{H}_{\text {norm }}}{\underset{\mathcal{H}_{\text {ext }}}{\gtrless}} \lambda_{2}\left(\hat{\theta}_{\text {ext }}\right)
$$

where $\hat{\theta}_{\text {norm }}$ and $\hat{\theta}_{\text {ext }}$ are the locations where $\lambda_{1}(\tilde{\theta})$ and $\lambda_{2}(\tilde{\theta})$ attain their global maximum, respectively. The synchronization parameters are eventually retrieved as indicated in (31) upon letting $\hat{\ell}=0$ in the expression $\hat{\varepsilon}=\varphi_{1}\left(\hat{\theta}_{\text {norm }}, \hat{\ell}\right) /(2 \pi)$ under the hypothesis $\mathcal{H}_{\text {norm }}$. Using the same arguments of Sect. IV.D, it turns out that the processing requirement of MLO in the absence of any irregular OFDM symbol reduces to $12 Q+$ 49 flops. It is worth noting that MLO can also be extended to communication systems where the CP size is chosen among $N_{H}>2$ available alternatives.

\section{Simulation Results}

\section{A. Simulation model}

The proposed synchronization scheme is applied to an LTE system compliant with the 3 GPP specifications [1] and operating in the $2.6 \mathrm{GHz}$ band. Recalling that the subcarrier distance in the LTE is fixed to $15 \mathrm{kHz}$, the signal bandwidth $B$ depends on the number of modulated subcarriers. In our study, we concentrate on the most popular LTE transmission modes with $B=5,10$ and $20 \mathrm{MHz}$. In any case, the received baseband signal is sampled with frequency $f_{s}=30.72 \mathrm{MHz}$ so that, irrespective of the transmission mode, the useful part of each OFDM symbol is composed by 2048 samples. To demonstrate the capability of the MLO in the presence of multipath distortions, we adopt the Extended Typical Urban (ETU) channel model as a propagation scenario. Hence, the channel response is characterized by 9 multipath components with maximum excess delay $\tau_{\max }=5 \mu$ s and statistically independent path gains following a zero-mean Gaussian distribution (Rayleigh fading). At each simulation run the synchronization parameters $(\theta, \varepsilon)$ are kept fixed since their value does not impact on the estimation's accuracy. In the normal CP mode, the index $\ell$ specifying the position of the irregular OFDM symbol is extracted from the set $\{0,1, \ldots, Q\}$ with equal probability.

\section{B. Performance evaluation}

The ability to detect the $\mathrm{CP}$ size is assessed through the probability of failure $P_{f}$, which represents the probability of taking the wrong decision between the two hypotheses $\mathcal{H}_{\text {norm }}$ and $\mathcal{H}_{\text {ext }}$. Fig. 2 illustrates $P_{f}$ conditioned to $\mathcal{H}_{\text {norm }}$ or $\mathcal{H}_{\text {ext }}$, i.e., $\operatorname{Pr}\left\{\hat{\mathcal{H}}=\mathcal{H}_{\text {ext }} \mid \mathcal{H}_{\text {norm }}\right\}$ or $\operatorname{Pr}\left\{\hat{\mathcal{H}}=\mathcal{H}_{\text {norm }} \mid \mathcal{H}_{\text {ext }}\right\}$, as a function of the signal-to-noise ratio (SNR). The signal bandwidth is $10 \mathrm{MHz}$ and $Q$ is set to 3 . The curves labeled GA are obtained by applying the Golnari Algorithm presented in [16] which, to the best of our knowledge, is the most powerful scheme available in the literature for $\mathrm{CP}$ size detection in the LTE downlink. As is seen, the best performance is achieved when the extended $\mathrm{CP}$ mode is in use. The reason is that the metric employed by both MLO and GA is based on the $N$-lag correlations between the $\mathrm{CP}$ and the last part of the OFDM symbol. In the extended $\mathrm{CP}$ mode, the correlations are averaged over a larger time window compared to the normal CP mode case, thereby increasing the resilience against thermal noise and channel impairments. Inspection of Fig. 2 indicates that MLO largely outperforms the GA and can work satisfactorily even at very low SNR values. In particular, at an SNR of $3 \mathrm{~dB}$ and in the normal CP mode, the probability of making a wrong decision with MLO reduces by approximately a factor of 100 with respect to GA. Since updating the GA metrics requires only 24 flops for each new received sample, it is fair to say that the advantage of MLO with respect to GA comes at the price of an increase of the processing load by nearly a factor of $2 Q+3 / 2$. Nevertheless, two major points need to be taken into account: 1) the MLO algorithm exhibits gains of several $\mathrm{dBs}$ with respect to the existing literature and these gains, mostly when a key operation like $\mathrm{CP}$ estimation is in play, may, in terms of benefits, offset the extra computational burden; 2) by the Moore's law the chip 
capacity approximately doubles every 18-24 months making certain processing issues less relevant with the passing of time.

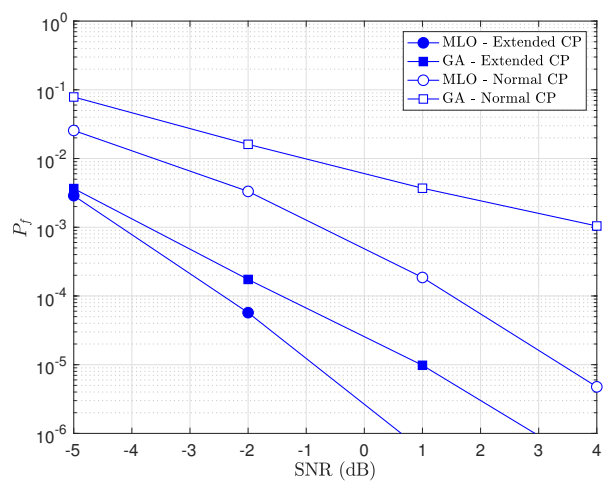

Fig. 2. Probability of failure vs. SNR for $Q=3$ and $B=10 \mathrm{MHz}$.

The accuracy of the timing estimates provided by the considered schemes is reported in Fig. 3. Extensive measurements indicate that both MLO and GA are biased as a consequence of the multipath dispersion, with the average error $\mathrm{E}\{\hat{\theta}-\theta\}$ being approximately equal to the root-mean-square (rms) channel delay spread. For this reason, we use the standard deviation of the timing estimate $\operatorname{Std}(\hat{\theta})=\sqrt{\mathrm{E}\left\{[\hat{\theta}-\mathrm{E}\{\hat{\theta}\}]^{2}\right\}}$ as a performance indicator, where $\mathrm{E}\{\hat{\theta}\}$ is numerically computed during the simulation. The results of Fig. 3 indicate that GA performs similarly to MLO in the extended CP mode, while a remarkable performance degradation is observed when the normal CP is adopted. Such a loss is a consequence of large timing errors (outliers) that occur whenever the $\mathrm{CP}$ size is incorrectly detected. Since GA exhibits a non-negligible probability of failure in the normal $\mathrm{CP}$ mode, it is more exposed to outliers than MLO, thereby leading to an enhanced variance of the timing estimates. This interpretation is validated by the results of Fig. 4, which illustrates the timing error measured under the hypothesis $\mathcal{H}_{\text {norm }}$ during 10,000 simulation trials with $Q=3, B=10 \mathrm{MHz}$ and $\mathrm{SNR}=4 \mathrm{~dB}$. Six outliers are clearly evident when GA is in use, while no outliers are observed with MLO in the same operating conditions. On the other hand, from Fig. 2 we see that in the extended CP mode the value of $P_{f}$ with both MLO and GA is remarkably smaller than in the normal CP case. Accordingly, outliers tend to disappear and only a marginal difference remains between the timing accuracy of the considered schemes. It is worth mentioning that large values of $\operatorname{Std}(\hat{\theta})$ in the normal CP mode reported in Fig. 3 at very low SNRs are due to the presence of outliers with both schemes.

Fig. 5 illustrates the mean square error of the frequency estimates, defined as $\operatorname{MSE}(\hat{\varepsilon})=\mathrm{E}\left\{(\hat{\varepsilon}-\varepsilon)^{2}\right\}$. The simulation set-up is the same as in Figs. 2 and 3, with $Q=3$ and $B=10$ $\mathrm{MHz}$. Comparing these results with the timing accuracy shown in Fig. 3, we see that the loss of GA with respect to MLO is greatly reduced in the normal $\mathrm{CP}$ mode, and totally disappears with the extended CP. We can explain such a behaviour by observing that, even in the presence of an outlier, the FFO error is restricted to the interval $[-1 / 2,1 / 2)$, so that its impact on the MSE is not as catastrophic as for the timing accuracy. For

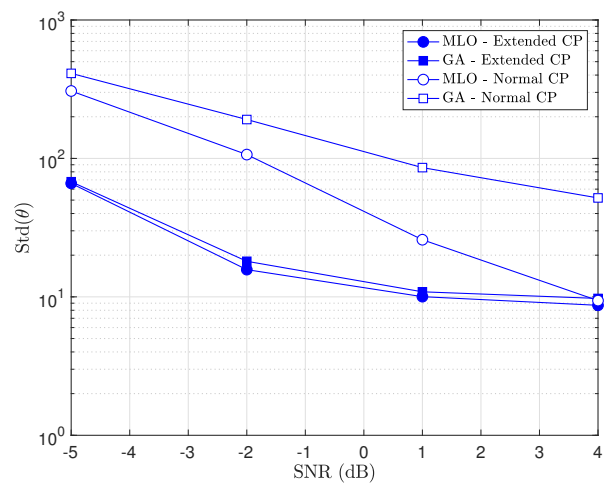

Fig. 3. Standard deviation of the timing estimates vs. SNR for $Q=3$ and $B=10 \mathrm{MHz}$.
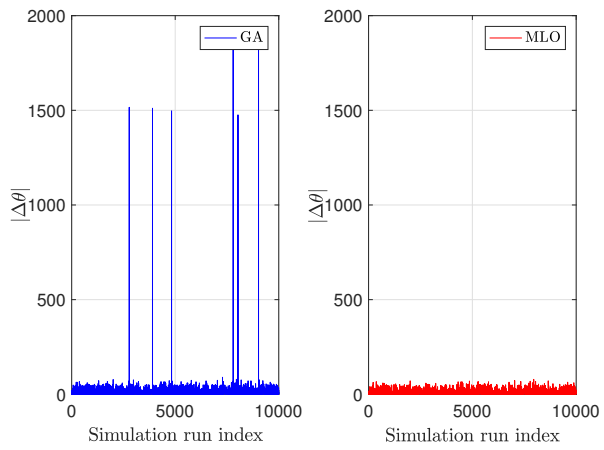

Fig. 4. Timing errors under the hypothesis $\mathcal{H}_{\text {norm }}$ with $Q=3, B=10$ $\mathrm{MHz}$ and $S N R=4 \mathrm{~dB}:$ a) GA and b) MLO.

example, with a failure probability of $5 \cdot 10^{-3}$ and assuming that the average FFO error generated by an outlier event is in the order of $|\hat{\varepsilon}-\varepsilon|=0.25$, the impact on $\operatorname{MSE}(\hat{\varepsilon})$ is approximately $3 \cdot 10^{-4}$. Since such a value is comparable to the accuracy of MLO under the $\mathcal{H}_{\text {norm }}$ hypothesis, we conclude that the occurrence of an outlier does not result into a remarkable increase of $\operatorname{MSE}(\hat{\varepsilon})$. Our investigation also indicates that, upon discarding all the failure events where the $\mathrm{CP}$ size is incorrectly detected, the accuracy of the timing and frequency estimates becomes practically the same with both MLO and GA. The reason is that, in such a genie-aided situation, the GA boils down to the classical synchronization algorithm derived in [6] for ML timing and FFO recovery over an AWGN channel. Hence, once the $C P$ mode has been successfully detected, the GA is expected to provide nearly optimum timing and frequency estimates, apart for a hypothetical loss arising from the presence of the irregular OFDM symbol in the LTE slot which, differently from MLO, is not accounted for by GA.

The impact of the observation length and signal bandwidth on the performance of MLO is assessed in Fig. 6, where $P_{f}$ is shown as a function of $Q$ at an SNR value of $0 \mathrm{~dB}$ and for $B=5,10$ and $20 \mathrm{MHz}$. It turns out that the performance improves as $Q$ grows large due to the increased length of the observation window. On the other hand, recalling that the received baseband signal in the LTE is sampled at a fixed frequency $f_{s}=30.72 \mathrm{MHz}$, the time-domain samples 


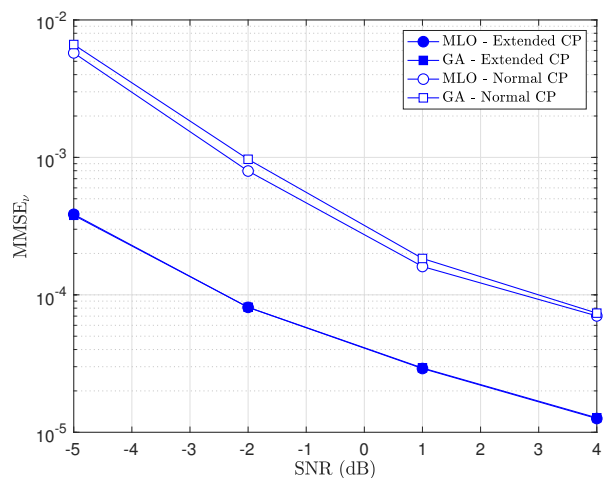

Fig. 5. Mean square error of the frequency estimates vs. SNR for $Q=3$ and $B=10 \mathrm{MHz}$.

become more and more correlated as the signal bandwidth reduces, with a corresponding deterioration of the capability to recognize the correct $\mathrm{CP}$ length. Such intuition is corroborated by the results of Fig. 6, where $P_{f}$ increases by approximately a factor of 25 when $B$ reduces from 20 to $5 \mathrm{MHz}$ and $Q \geq 3$.

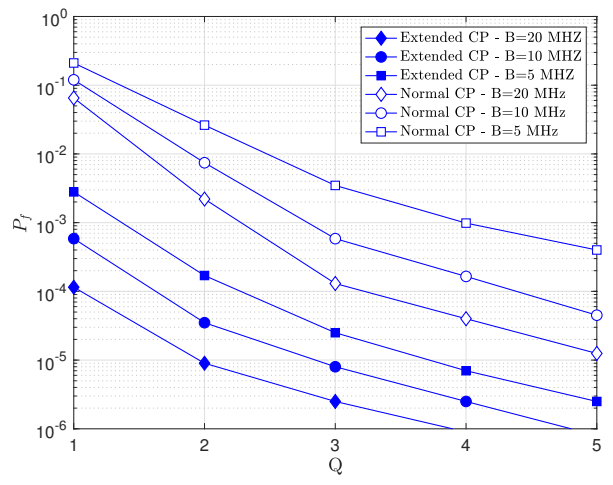

Fig. 6. Probability of failure vs. $Q$ with $\mathrm{SNR}=0 \mathrm{~dB}$ and $B=5,10$ and 20 MHz.

Figs. 7 and 8 illustrate $\operatorname{Std}(\hat{\theta})$ and $\operatorname{MSE}(\hat{\varepsilon})$, respectively, as a function of $Q$ in the same operating scenario of Fig. 6. Again, we see that the accuracy of the recovered synch parameters improves with the signal bandwidth and observation length except for the timing estimate which, in the extended $\mathrm{CP}$ mode, exhibits an irreducible floor as $Q$ grows large. Such a floor is a consequence of the different channel snapshots generated throughout the numerical simulations, which are characterized by different delay spreads. Hence, even in the presence of a very long observation window, the estimate $\hat{\theta}$ varies at each simulation run depending on the actual channel realization. This results into a non-zero value of the measured quantity $\hat{\theta}-\mathrm{E}\{\hat{\theta}\}$ and ultimately leads to a floor in $\operatorname{Std}(\hat{\theta})$.

\section{CONCLUSIONS}

We have presented a new method for joint $\mathrm{CP}$ size detection and timing/frequency synchronization in the downlink of an LTE communication system. The proposed algorithm (MLO) is based on ML arguments and exploits the time-domain correlation between the $\mathrm{CP}$ and the last part of the OFDM symbol.

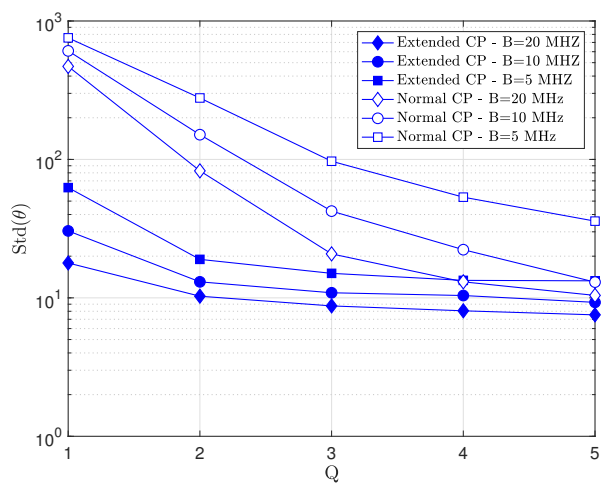

Fig. 7. Standard deviation of the timing estimates vs. $Q$ with $\mathrm{SNR}=2 \mathrm{~dB}$ and $B=5,10$ and $20 \mathrm{MHz}$.

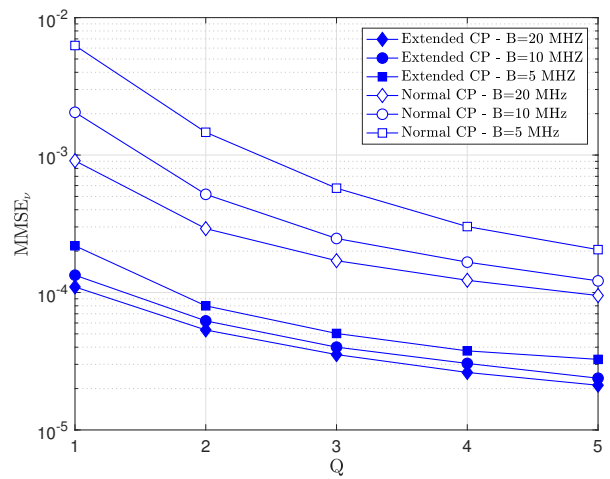

Fig. 8. Mean square error of the timing estimates vs. $Q$ with $\mathrm{SNR}=2 \mathrm{~dB}$ and $B=5,10$ and $20 \mathrm{MHz}$.

Once the CP length has been detected, timing and frequency recovery is accomplished with methods similar to the classical approach employed in OFDM transmissions. Although it is derived under the assumption that channel distortion only consists of additive noise, MLO is found to perform well even in a highly dispersive propagation environment characterized by a large delay spread. Some indication is provided as how to extend the application of MLO to future OFDM systems with adaptive $\mathrm{CP}$ size selection.

Numerical results indicate that, compared to alternative schemes available in the literature, our method exhibits improved estimation accuracy and enhanced detection capability at the price of an increased computational load. The penalty in terms of required flops is justified by the fact that MLO can operate at low SNR values, where other competing schemes derived in a heuristic fashion are characterized by poor performance. Furthermore, the improved ability to correctly detect the $\mathrm{CP}$ size allows one to reduce the length of the observation window, which is essential for fast detection of neighboring cells and fast handover operations.

\section{APPENDIX A: DERIVATION OF (13)}

The covariance matrix $\mathbf{C}_{x}$ in (9) can be expressed as

$$
\mathbf{C}_{x}=\boldsymbol{\Gamma}(\nu) \mathbf{C} \boldsymbol{\Gamma}^{H}(\nu)
$$

where $\mathbf{C}=\mathbf{C}_{s}+\sigma_{w}^{2} \mathbf{I}_{M}$. In this appendix we derive the expressions of $\operatorname{det}(\mathbf{C})$ and $\mathbf{C}^{-1}$, which are central in the 
derivation of (13). For space limitations, we focus on the case $\ell=0$ since the other cases can be treated with a similar analysis.

- Determinant of $\mathbf{C}$. The matrix $\mathbf{C}$ is by construction blockdiagonal, i.e.,

$$
\mathbf{C}=\operatorname{diag}\left\{\mathbf{C}_{1}, \mathbf{C}_{2}, \ldots, \mathbf{C}_{Q}, \mathbf{C}_{Q+1}\right\}
$$

where $\mathbf{C}_{i}=\mathbf{C}_{z_{i}}+\sigma_{w}^{2} \mathbf{I}_{N+L_{1}}$ for $i=1 \ldots, Q$ and $\mathbf{C}_{Q+1}=\mathbf{C}_{\bar{z}_{Q+1}}+\sigma_{w}^{2} \mathbf{I}_{Q\left(L_{2}-L_{1}\right)}$. It follows that

$$
\operatorname{det} \mathbf{C}=\prod_{i=1}^{Q+1} \operatorname{det} \mathbf{C}_{i}
$$

From (12) and remembering that $\sigma^{2}=\sigma_{s}^{2}+\sigma_{w}^{2}$, the matrix $\mathbf{C}_{i}(1 \leq i \leq Q)$ can be written as

$$
\mathbf{C}_{i}=\left[\begin{array}{cc}
\sigma^{2} \mathbf{I}_{N} & \mathbf{B}^{T} \\
\mathbf{B} & \sigma^{2} \mathbf{I}_{L_{1}}
\end{array}\right]
$$

where $\mathbf{B}=\left[\sigma_{s}^{2} \mathbf{I}_{L_{1}}, \mathbf{0}_{L_{1}, N-L_{1}}\right]$. Accordingly, exploiting the block structure in (A.4), one obtains

$$
\operatorname{det} \mathbf{C}_{i}=\operatorname{det}\left(\sigma^{2} \mathbf{I}_{N}-\frac{1}{\sigma^{2}} \mathbf{B}^{T} \mathbf{B}\right) \operatorname{det}\left(\sigma^{2} \mathbf{I}_{L_{1}}\right)
$$

Since it is

$$
\left(\sigma^{2} \mathbf{I}_{N}-\frac{1}{\sigma^{2}} \mathbf{B}^{T} \mathbf{B}\right)=\left[\begin{array}{cc}
\left(\sigma^{2}-\frac{\sigma_{S}^{4}}{\sigma^{2}}\right) \mathbf{I}_{L_{1}} & \mathbf{0} \\
\mathbf{0} & \sigma^{2} \mathbf{I}_{N-L_{1}}
\end{array}\right],
$$

the determinant in (A.5) can be computed as

$\operatorname{det} \mathbf{C}_{i}=\left(\sigma^{2}-\frac{\sigma_{S}^{4}}{\sigma^{2}}\right)^{L_{1}}\left(\sigma^{2}\right)^{N-L_{1}}\left(\sigma^{2}\right)^{L_{1}}=\left(1-\gamma^{2}\right)^{L_{1}}$

where $\gamma=\frac{\sigma_{S}^{2}}{\sigma^{2}}$. Replacing (A.7) in (A.3) and considering that $\mathbf{C}_{Q+1}=\sigma^{2} \mathbf{I}_{Q\left(L_{2-L 1}\right)}$, we can write

$$
\operatorname{det} \mathbf{C}=\left(1-\gamma^{2}\right)^{Q L_{1}}\left(\sigma^{2}\right)^{M}
$$

\section{- Inverse of $\mathbf{C}$.}

Acknowledging the block-diagonal structure of the correlation matrix $\mathbf{C}$, its inverse is

$$
\mathbf{C}^{-1}=\operatorname{diag}\left\{\mathbf{C}_{1}^{-1}, \mathbf{C}_{2}^{-1}, \ldots, \mathbf{C}_{Q}^{-1}, \mathbf{C}_{Q+1}^{-1}\right\},
$$

where $\mathbf{C}_{i}^{-1}(i=1,2, \ldots, Q)$ can be computed as

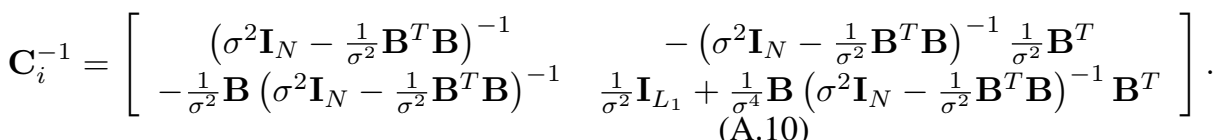

Accordingly, since it is

$$
\left(\sigma^{2} \mathbf{I}_{N}-\frac{1}{\sigma^{2}} \mathbf{B}^{T} \mathbf{B}\right)^{-1}=\frac{1}{\sigma^{2}}\left[\begin{array}{cc}
\frac{1}{1-\gamma^{2}} \mathbf{I}_{L_{1}} & \mathbf{0}_{L_{1}, N-L_{1}} \\
\mathbf{0}_{N-L_{1}, L_{1}} & \mathbf{I}_{N-L_{1}}
\end{array}\right],
$$

the inverse of a diagonal block $\mathbf{C}_{i}$ is found to be

$$
\mathbf{C}_{i}^{-1}=\frac{1}{\sigma^{2}}\left[\begin{array}{ccc}
\frac{1}{1-\gamma^{2}} \mathbf{I}_{L_{1}} & \mathbf{0}_{L_{1}, N-L_{1}} & -\frac{\gamma}{1-\gamma^{2}} \mathbf{I}_{L_{1}} \\
\mathbf{0}_{N-L_{1}, L_{1}} & \mathbf{I}_{N-L_{1}} & \mathbf{0}_{N-L_{1}, L_{1}} \\
-\frac{\gamma}{1-\gamma^{2}} \mathbf{I}_{L_{1}} & \mathbf{0}_{L_{1}, N-L_{1}} & \frac{1}{1-\gamma^{2}} \mathbf{I}_{L_{1}}
\end{array}\right]_{(\mathrm{A} .12)}
$$

Now, replacing in (10) the expressions found in (A.8), (A.9) and (A.12), and keeping in mind that $\mathbf{C}_{Q+1}=\sigma^{2} \mathbf{I}_{Q\left(L_{2}-L_{1}\right)}$, yields (13).

\section{REFERENCES}

[1] 3GPP TS 36.211 V10.4.0: Evolved Universal Terrestrial Radio Access (E-UTRA); Physical Channels and Modulation (Release 10), 2011-12.

[2] I. T. S. Sesia and M. Baker, LTE-the UMTS Long Term Evolution: from theory to practice, New York: John Wiley \& Sons, 2009.

[3] Y. Tsai, G. Zhang, D. Grieco, F. Ozluturk, and X. Wang, "Cell search in 3GPP long term evolution systems", IEEE Veh. Technol. Mag., pp. 23-29, June 2007.

[4] M. Morelli and M. Moretti, "A robust maximum likelihood scheme for PSS detection and integer frequency offset recovery in LTE systems," IEEE Trans. Wireless Commun., vol. 15, no. 2, Feb. 2016.

[5] M. Morelli and M. Moretti, "A maximum likelihood approach for SSS detection in LTE systems," IEEE Trans. Wireless Commun., vol. 16, no. 4, Apr. 2017.

[6] J.-J. van de Beek, M. Sandell, and P. O. Börjesson, "ML estimation of time and frequency offset in OFDM systems", IEEE Trans. on Signal Processing, vol. 45, n. 7, pp. 1800-1805, July 1997.

[7] K. Manolakis, D. M. G. Estévez, V. Jungnickel, W. Xu and C. Drewes, "A closed concept for synchronization and cell search in 3GPP LTE systems", in Proc. of Wireless Commun. and Net. Conf. (WCNC) 2009, Budapest, Apr. 2009

[8] D. B. Lin, J. C. Hsieh, and H. P. Lin, "Improved joint correlated detection in cell search and synchronization procedure in 3GPP LTE downlink system", in Proc. of 2012 Int. Conf. on Anti-Counterfeiting, Security and Identification (ASID), Aug. 2012.

[9] X. Yang, Y. Xiong, G. Jia, W. Fang, and X. Zheng, "PSS based time synchronization for 3GPP LTE downlink receivers", in Proc. of 13th Int. Conf. on Commun. Techn. (ICCT) 2011, pp. 930-933, 2011.

[10] Y. Yu and Q. Zhu, "A novel time synchronization for 3 GPP LTE cell search", in Proc. of 8th Int. Conf. on Commun. and Net. in China, 2013.

[11] S. Huang, Y. Su, Y. He, and S. Tang, "Joint time and frequency offset estimation in LTE downlink", in Proc. of 7th International ICST Conference on Communications and Networking in China (CHINACOM), pp. 394-398, 2012.

[12] 3GPP TSG RAN WG1 LTE TDD Ad hoc R1-071884, "P-SCH/S-SCH design in alternative TDD frame structure", Samsung, Apr. 2007.

[13] J.-In Kim, J.-Su Han, H.-J. Roh, and H.-J. Choi, "SSS detection method

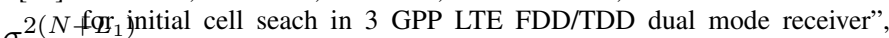
in Proc. of 9th Int. Symp. on Commun. and Inf. Techn. (ISCIT), pp. 199-203, 2009.

[14] K. Pushpa, Ch. N. Kishore, and Y. Yoganandam, "Estimation of frequency offset, cell ID and CP length in OFDMA mode of WMAN", in Proc. of 2008 IEEE Region Conference (TENCON), 2008.

[15] Y. Kim and K. Chang, "Complexity optimized CP length pre-decision metric for cell searcher in the downlink of 3GPP LTE system", in Proc. of IEEE 20th International Symposium on Personal, Indoor and Mobile Radio Communications (PIMRC), pp. 895-899, 2009.

[16] A. Golnari, M. Shabany, A. Nezamalhosseini, and G. Gulak, "Design and implementation of time and frequency synchronization in LTE", IEEE Trans. on Very Large Scale Integration (VLSI) Systems, vol. 23, n. 12, pp. 2970-2982, Dec. 2015.

[17] S. M. Kay, Fundamentals of Statistical Signal Processing: Estimation Theory, Prentice Hall, Englewood Cliffs, New Jersey, 1993. 